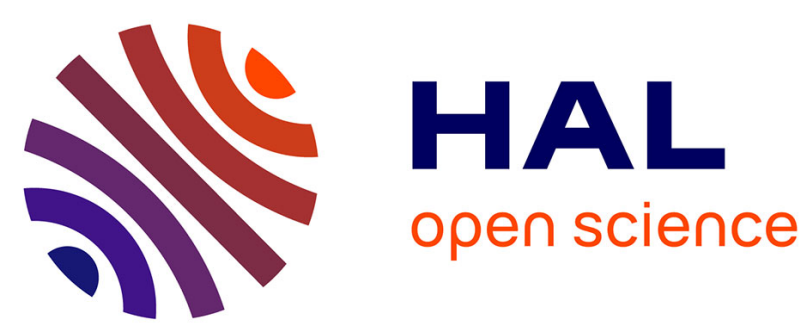

\title{
The Cayley Grassmannian
}

Laurent Manivel

\section{To cite this version:}

Laurent Manivel. The Cayley Grassmannian. Journal of Algebra, 2018, 10.1016/j.jalgebra.2018.02.010 . hal-01269989

HAL Id: hal-01269989

https://hal.science/hal-01269989

Submitted on 5 Feb 2016

HAL is a multi-disciplinary open access archive for the deposit and dissemination of scientific research documents, whether they are published or not. The documents may come from teaching and research institutions in France or abroad, or from public or private research centers.
L'archive ouverte pluridisciplinaire HAL, est destinée au dépôt et à la diffusion de documents scientifiques de niveau recherche, publiés ou non, émanant des établissements d'enseignement et de recherche français ou étrangers, des laboratoires publics ou privés. 


\title{
The Cayley Grassmannian
}

\author{
Laurent MANIVEL
}

February 2016

\begin{abstract}
We study the projective variety $C G$ parametrizing four dimensional subalgebras of the complex octonions, which we call the Cayley Grassmannian. We prove that it is a spherical $G_{2}$-variety with only three orbits that we describe explicitely. Its cohomology ring has a basis of Schubert type classes and we determine the intersection product completely.
\end{abstract}

\section{Introduction}

There exists only four real normed algebras up to isomorphism: the algebras of real and complex numbers, the Hamilton algebra of quaternions and the Cayley algebra of octonions. The Cayley-Dickson doubling process allows to construct these algebras iteratively as a chain

$$
\mathbb{R} \subset \mathbb{C} \subset \mathbb{H} \subset \mathbb{O} .
$$

The Cayley algebra $\mathbb{O}$ contains lots of subalgebras isomorphic to the Hamilton algebra. In fact its automorphism group $G_{2}=A u t(\mathbb{O})$ acts transitively on the set of four dimensional subalgebras, which has a natural structure of compact manifold isomorphic with the homogeneous space

$$
\mathrm{G}_{2} / \mathrm{SO}_{3} \times \mathrm{SO}_{3}
$$

The main goal of this note is to describe in some details what happens over the complex numbers, that is, when we consider the complexified algebra of octonions. The set of four dimensional subalgebras of the complexified algebra of octonions is a closed subvariety of the complex Grassmannian $G(4,8)$. Since all these subalgebras contain the unit element, we can focus on their imaginary part, which is parametrized by a closed subvariety $C G$ of the Grassmannian $G(3,7)$. An important difference with the real case is that the action of $G_{2}$ is no longer transitive. We will prove the following statement.

Theorem 1.1 The variety $C G$ is a smooth irreducible variety of dimension eight. The action of $G_{2}$ on $C G$ is spherical, in particular quasi-homogeneous, and has only three orbits. 
A maximal torus in $G_{2}$ has fifteen fixed points in $C G$. There are infinitely many invariant curves joining these points but we can nevertheless determine the equivariant cohomology ring of $C G$ by localization to the fixed points. Then we can easily derive the usual cohomology ring, which is isomorphic to the Chow ring.

Interestingly the Betti numbers of $C G$ are the same as those of the Grassmannian $G(2,6)$, and the presentation of the Chow ring that we obtain is quite similar. In particular the Picard number of $C G$ is one. We thus get a minimal smooth compactification of the eight dimensional affine space, whose boundary we describe in some detail.

Our description of the Chow ring is the following. Denote by $\sigma_{1}$ the hyperplane section. There is a Schubert type class $\sigma_{2}$, of degree two, such that:

Theorem 1.2 The rational cohomology ring of $C G$ is

$$
H^{*}(C G, \mathbb{Q})=\mathbb{Q}\left[\sigma_{1}, \sigma_{2}\right] /\left\langle\sigma_{1}^{5}-5 \sigma_{1}^{3} \sigma_{2}+6 \sigma_{1} \sigma_{2}^{2}, 16 \sigma_{2}^{3}-27 \sigma_{1}^{2} \sigma_{2}^{2}+9 \sigma_{1}^{4} \sigma_{2}\right\rangle .
$$

The Picard group of $C G$ is cyclic, and the degree of $C G$ with respect to the (very) ample generator of the Picard group is 182 . We compute the generators of its homogeneous coordinate ring thanks to the structure of its orbit closures. We also compute the Chern classes of $C G$, and deduce that its projective dual is a hypersurface of degree 17 in $\mathbb{P}^{28}$.

In a subsequent paper we will compute the quantum cohomology of $C G$, which turns out to be semisimple. We also plan to describe its derived category which, if the the Dubrovin conjecture is correct, should be generated by an exceptional collection.

More generally, it would be extremely interesting to extend the huge amount of information that we have about the quantum cohomology and the derived category of homogeneous spaces (although the picture is not complete yet) to the more general setting of quasi-homogeneous, or more specifically, spherical varieties. Beyond the intrisic interest and beauty of the Cayley Grassmannian, we consider the present study as a tiny piece of this more general program.

Acknowledgements. This paper was written somewhere between the CRM (Montreal University) and the CIRGET (UQAM), during the Fall of 2014. It is a pleasure to thank these institutions for the excellent working conditions and the stimulating environments. Many thanks also to Steven Lu, Steven Boyer and Laurent Habsieger for their hospitality.

\section{Four dimensional subalgebras of $\mathbb{O}$}

\subsection{A reminder about $G_{2}$}

From now on we work exclusively over the field over complex numbers. We still denote by $\mathbb{O}$ the algebra of octonions with complex coefficients. We will need two different points of views over the smallest of the exceptional complex simple Lie groups. 


\subsection{1 $G_{2}$ and the octonions}

One can describe $G_{2}$ as the automorphism group of the Cayley algebra of octonions, $G_{2}=\operatorname{Aut}(\mathbb{O})$ (we use [Baez] as a convenient reference about the octonions). In particular it preserves the unit element $e_{0}=1$, the multiplicative norm $q$, and therefore the space of imaginary octonions $\operatorname{Im}(\mathbb{O})=e_{0}^{\perp}$.

The multiplication table of the octonions can be encoded in the oriented Fano plane, whose vertices are in correspondence with the vectors $e_{1}, \ldots, e_{7}$ of an orthonormal basis of the imaginary octonions.

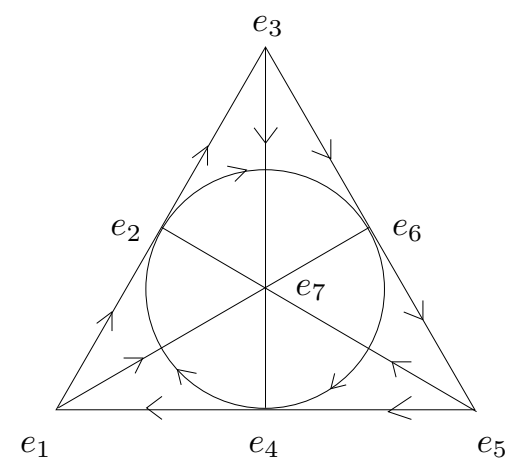

1. The multiplication table of the octonions

This plane is made of seven lines (including the central circle) passing through seven points each. In order to multiply $e_{i}$ with $e_{j}$ for $i \neq j$, we need to find the line to which they both belong, and then $e_{i} e_{j}= \pm e_{k}$ for $e_{k}$ the third point on this line; the sign is + if the (cyclic) order $i j k$ of the vertices provides the correct orientation of the line, and - otherwise. Finally $e_{i}^{2}=-1$ for any $i \neq 0$.

The previous picture also defines a skew-symmetric three-form, given as the sum of the contributions of the seven (cyclically oriented) lines in the plane (note that the norm identifies $\operatorname{Im}(\mathbb{O})$ with its dual):

$$
\Omega=\sum_{\text {lines }(i j k)} e_{i} \wedge e_{j} \wedge e_{k} .
$$

In terms of the octonionic multiplication, this can be written as

$$
\Omega=\frac{1}{6} \sum_{i j} e_{i} \wedge e_{j} \wedge e_{i} e_{j} .
$$

\subsubsection{The algebraic group $G_{2}$}

Recall that the algebraic group $G_{2}$ is a simply connected group of rank two and dimension fourteen. One of its fundamental representations is the adjoint representation on the Lie algebra $\mathfrak{g}_{2}$, which is encoded in the root system also denoted $G_{2}$ : 


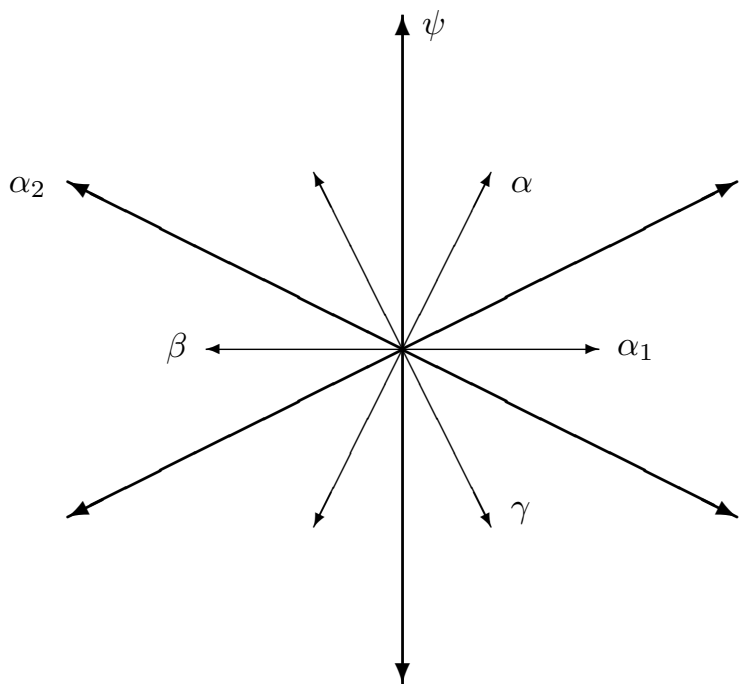

2. The root system of type $G_{2}$

This root system is made of two root systems of type $A_{2}$, formed by the short and the long roots respectively. The highest weight of the adjoint representation is the highest root $\psi=3 \alpha_{1}+2 \alpha_{2}$.

The other fundamental representation is generated by the short roots. Its highest weight is the highest short root $\alpha=2 \alpha_{1}+\alpha_{2}$; its weights are the six short roots and zero, all with multiplicity one, so that its dimension is seven; we denote this representation by $V_{7}$. The action of $G_{2}$ on $V_{7}$ preserves a non degenerate quadratic form $q$, and also a skew-symmetric three form $\Omega$. (In fact we can define $G_{2}$ exactly that way: $\wedge^{3} V_{7}^{*}$ is a prehomogeneous vector space under the action of $G L_{7}$, and the stabilizer in $G L_{7}$ of a generic element $\Omega$ is a copy of $G_{2}$.) The quadratic form $q$ and the skew-symmetric three form $\Omega$ can be related through the following relation (where the choice of the non zero constant $c$ is arbitrary):

$$
q(x) \Theta=c i(x) \Omega \wedge i(x) \Omega \wedge \Omega,
$$

where $i(x)$ is the contraction operator with $x \in V_{7}$, and $\Theta$ is a generator of $\wedge^{7} V_{7}^{*}$.

Consider the decomposition into weight spaces

$$
V_{7}=\mathbb{C} u_{0} \oplus \mathbb{C} u_{\alpha} \oplus \mathbb{C} u_{-\alpha} \oplus \mathbb{C} u_{\beta} \oplus \mathbb{C} u_{-\beta} \oplus \mathbb{C} u_{\gamma} \oplus \mathbb{C} u_{-\gamma} .
$$

Here we denoted the six short roots of $G_{2}$ as $\pm \alpha, \pm \beta, \pm \gamma$, with $\alpha+\beta+\gamma=0$, in order to enhance the natural $S_{3}$-symmetry. The vector $u_{0}$ has non zero norm, the other weight vectors are isotropic and we can normalize the octonionic quadratic form so that its expression in the dual basis is

$$
q=v_{0}^{2}+v_{\alpha} v_{-\alpha}+v_{\beta} v_{-\beta}+v_{\gamma} v_{-\gamma} .
$$


The invariant three-form $\Omega$ up to scalar can then be normalized as

$\Omega=v_{0} \wedge v_{\alpha} \wedge v_{-\alpha}+v_{0} \wedge v_{\beta} \wedge v_{-\beta}+v_{0} \wedge v_{\gamma} \wedge v_{-\gamma}+v_{\alpha} \wedge v_{\beta} \wedge v_{\gamma}+v_{-\alpha} \wedge v_{-\beta} \wedge v_{-\gamma}$

In order to reconcile the two approaches, note that $\operatorname{Im}(\mathbb{O})=V_{7}$, the invariant norm being the restriction of the multiplicative norm. Moreover if in the previous expression of $\Omega$, we let $v_{\alpha}=e_{\alpha}+e_{-\alpha}, v_{-\alpha}=e_{\alpha}-e_{-\alpha}$, and so on, it becomes a sum of seven terms that define a plane projective geometry. Moreover the octonionic product can be recovered directly from $\Omega$. Indeed, note that the induced map from $V_{7} \times V_{7}$ to $V_{7}$ sending $(x, y)$ to $\operatorname{Im}(x y)$ is skew-symmetric; in terms of the three-form,

$$
\operatorname{Im}(x y)=\iota(y \wedge x) \Omega .
$$

A last remark is in order. Consider the two fundamental representations, and their projectivizations: each of them contains a unique closed $G_{2}$-orbit. In $\mathbb{P}\left(V_{7}\right)$ this is simply the quadric $\mathbb{Q}^{5}$ defined by the norm. In $\mathbb{P}\left(\mathfrak{g}_{2}\right)$ this is by definition the adjoint variety of $G_{2}$, which we denote by $G_{2}^{a d}$ and is also five dimensional. Since $\mathfrak{g}_{2} \subset \mathfrak{s o}_{7}=\wedge^{2} V_{7}$, the adjoint variety is a subvariety of the Grassmannian $G\left(2, V_{7}\right)$. It was proved in [LM1] that the adjoint variety parametrizes the null-planes inside $V_{7}=\operatorname{Im}(\mathbb{O})$ : those on which the octonionic product is identically zero. In particular, the following statement will be useful.

Proposition 2.1 The action of $G_{2}=\operatorname{Aut}(\mathbb{O})$ is transitive

1. on the set of non zero isotropic imaginary octonions;

2. on the set of null-planes.

\subsection{Non degenerate subalgebras}

Let $\mathbb{A}=\mathbb{C} 1 \oplus \operatorname{Im}(\mathbb{A})$ be a four dimensional subalgebra of the complexified Cayley algebra.

Definition 2.2 We say that $\mathbb{A}$ is non degenerate when the restriction of the octonionic norm to $\mathbb{A}$ is a non degenerate quadratic form. Otherwise we say that $\mathbb{A}$ is degenerate.

Suppose that $\mathbb{A}$ is non degenerate. Choose a non isotropic vector $e_{1}$ in $\operatorname{Im}(\mathbb{A})$, and normalize it so that its norm is equal to one. Then $e_{1}^{2}=-e_{1} \overline{e_{1}}=$ -1 . Chose another norm one vector $e_{2}$ in $\operatorname{Im}(\mathbb{A})$, orthogonal to $e_{1}$. Then $e_{2}^{2}=-1$. Let $e_{3}=e_{1} e_{2}=-e_{2} e_{1}$. Recall that although the Cayley algebra is non associative, it is alternative, which means that any subalgebra generated by two elements is associative. Hence $e_{1} e_{3}=e_{1}\left(e_{1} e_{2}\right)=\left(e_{1} e_{1}\right) e_{2}=-e_{2}$ and similarly $e_{2} e_{3}=e_{1}$. Moreover, $1, e_{1}, e_{2}, e_{3}$ is a basis of $\mathbb{A}$. Indeed, if this was not the case we would have a relation $e_{3}=x+y e_{1}+z e_{2}$; multiplying by $e_{2}$ on the left and on the right we would get $e_{2}=x e_{1}-y-z e_{3}=-x e_{1}+y-z e_{3}$, hence $x=y=0$; multiplying by $e_{1}$ on the left and on the right we get similarly $x=z=0$; hence $e_{3}=0$, a contradiction since $e_{3}$ has norm one. 
This proves that $\mathbb{A}$ is isomorphic with the complexified Hamilton algebra. Moreover, since the norm is non degenerate on $\mathbb{A}$ as well as on $\mathbb{O}$, we can choose a norm one vector $e_{4}$ orthogonal to $\mathbb{A}$. Then letting $e_{5}=e_{3} e_{4}, e_{6}=e_{2} e_{4}$ and $e_{7}=-e_{1} e_{4}$, we can check that we get a basis $1, e_{1}, \ldots, e_{7}$ of $\mathbb{O}$ whose multiplication table is the standard one. For example, the Moufang identities imply that

$$
e_{5} e_{6}=-\left(e_{4} e_{3}\right)\left(e_{2} e_{4}\right)=-e_{4}\left(e_{3} e_{2}\right) e_{4}=e_{4} e_{1} e_{4}=e_{1},
$$

and so on. We get the following statement:

Lemma 2.3 The automorphism group of $\mathbb{O}$ acts transitively on the set of its four dimensional non degenerate subalgebras, which are all copies of the complexified Hamilton algebra.

Now we compute the stabilizer of a non degenerate subalgebra of $\mathbb{O}$, say $\mathbb{H}$.

Proposition 2.4 The stabilizer of $\mathbb{H}$ in $G_{2}=A u t(\mathbb{O})$ is the product $H=A_{1}^{s} A_{1}^{l}$ of the two copies of $S L_{2}$ in $G_{2}$ generated by its short roots and its long roots, respectively.

Proof. Let $H$ denote the stabilizer of $\mathbb{H}$ in $G_{2}$. It follows from the proof of the previous lemma that the map $r: H \rightarrow \operatorname{Aut}(\mathbb{H}) \simeq S L_{2}$ is surjective. Choosing a fixed $e_{4}$ we get a splitting $\operatorname{Aut}(\mathbb{H}) \hookrightarrow H$. Varying $e_{4}$ we see that the kernel $K$ of $r$ is in bijection with the space of unit vectors in $\mathbb{H}^{\perp}$, yielding another copy of $S L_{2}$. The fact that these two $S L_{2}$ correspond to $A_{1}^{s}$ and $A_{1}^{l}$ is straightforward. In fact we may suppose that $\operatorname{Im}(\mathbb{H})$ is generated by the three vectors $u_{0}, u_{\alpha}, u_{-\alpha}$. Then the (short) $A_{1}$ generated by $\alpha$ preserves $\mathbb{H}$ and acts non trivially on it, while the (long) $A_{1}$ generated by the orthogonal roots acts trivially on $\mathbb{H}$.

\subsection{Degenerate subalgebras}

Now suppose that $\mathbb{A}$ is degenerate.

Lemma 2.5 A contains a null plane $N$.

Proof. Let $x$ be a non zero vector in the kernel of the quadratic form. This means that $x \in \mathbb{A} \subset x^{\perp}$. In particular $x^{2}=-q(x)=0$. Moreover, since $\operatorname{Re}(x y)=q(x, \bar{y})$, the endomorphism of $\mathbb{A}$ defined by left multiplication by $x$ stabilizes $\operatorname{Im}(\mathbb{A})$. Its square is zero since $x^{2}=0$ and $\mathbb{O}$ is alternative. Therefore there exists $y$ in $\operatorname{Im}(\mathbb{A})$, independent of $x$, such that $x y=0$, hence also $y x=0$ since $x$ and $y$, being orthogonal, anticommute. Note that this forces $y$ to be isotropic, since multiplying $x y=0$ on the right by $\bar{y}=-y$ we get $q(y) x=0$. Hence $y^{2}=0$ and $N=\langle x, y\rangle$ is a null-plane.

Lemma 2.6 $\operatorname{Im}(\mathbb{A})$ is contained in $N^{\perp}$. Conversely, any three-dimensional subspace of $N^{\perp}$ containing $N$ is the imaginary part of a degenerate subalgebra of $\mathbb{O}$. 
Proof. Consider $z$ in $\operatorname{Im}(\mathbb{A})-N$. Then the non zero vector $x z$ belongs to the kernel of left multiplication by $x$ in $\mathbb{A}$. Since this kernel is $N$, there exist scalars $a$ and $b$ such that $x z=a x+b y$. Necessarily $b \neq 0$, otherwise we would get $(x-a 1) z=0$ and then $z=0$ since $x-a 1$ is not isotropic $(a \neq 0$ since $z$ is not in $N$ ). Hence $y$ is a combination of $x$ and $x z$, which easily implies that $y$ and $z$ anticommute. But this means they are orthogonal and we can conclude that $z$ belongs to $N^{\perp}$. So $\operatorname{Im}(\mathbb{A}) \subset N^{\perp}$.

In order to prove the second part of the statement we may use the transivity of $G_{2}$ on the set of null-planes. This allows us to suppose that $N=\langle x, y\rangle$ with $x=e_{1}+i e_{2}$ and $y=e_{6}+i e_{7}$. Then $N^{\perp}=N \oplus\left\langle e_{3}, e_{4}, e_{5}\right\rangle$ and an explicit computation yields

$$
\begin{aligned}
& x e_{3}=i x, \quad x e_{4}=i y, \quad x e_{5}=y, \\
& y e_{3}=-i y, \quad y e_{4}=i x, \quad y e_{5}=-x \text {. }
\end{aligned}
$$

This implies the claim.

Consider as above $z$ in $\operatorname{Im}(\mathbb{A})-N$. Since $z \in N^{\perp}$, we have only two possibilities. Either $z$ is not isotropic, so that the rank of the multiplicative norm on $\operatorname{Im}(\mathbb{A})$ is equal to one; in this case $N$ is the only null-space contained in $\mathbb{A}$. Either $z$ is isotropic, so that the multiplicative norm is identically zero on $\operatorname{Im}(\mathbb{A})$. But then the endomorphism of $\operatorname{Im}(\mathbb{A})$ defined by left multiplication by $z$ has again rank one, and by the same argument as above $z$ is contained in another null-plane $N^{\prime}$ of $\operatorname{Im}(\mathbb{A})$. Let $u$ be a generator of $N \cap N^{\prime}$. Then $u \cdot \operatorname{Im}(\mathbb{A})=0$. But the kernel of the left multiplication by $u$ on $\mathbb{O}$ is precisely $u \mathbb{O}$, which is a four dimensional isotropic subspace of $\mathbb{O}$. In particular it cannot be contained in $\operatorname{Im}(\mathbb{O})$ and we conclude that necessarily

$$
\operatorname{Im}(\mathbb{A})=u \mathbb{O} \cap \operatorname{Im}(\mathbb{O}),
$$

which is indeed the imaginary part of a subalgebra of $\mathbb{O}$.

We have finally classified the degenerate four dimensional subalgebras of $\mathbb{O}$.

Proposition 2.7 Let $\mathbb{A}$ be a degenerate four dimensional subalgebra of $\mathbb{O}$. Then:

1. either $\operatorname{Im}(\mathbb{A})$ is totally isotropic, there is a unique isotropic line $\ell$ such that $\operatorname{Im}(\mathbb{A})=\ell \mathbb{O} \cap \operatorname{Im}(\mathbb{O})$, and each plane in $\operatorname{Im}(\mathbb{A})$ containing $\ell$ is a null-plane,

2. $\operatorname{or} \operatorname{Im}(\mathbb{A})$ is not totally isotropic, it contains a unique null-plane $N$ and it is contained in $N^{\perp}$.

Up to the action of $G_{2}$, a four dimensional subalgebra of $\mathbb{O}$ is thus isomorphic to one of the following:

$$
\begin{aligned}
& H_{0}=\left\langle e_{0}, e_{1}, e_{2}, e_{3}\right\rangle \\
& H_{1}=\left\langle e_{0}, e_{1}+i e_{2}, e_{6}+i e_{7}, e_{3}\right\rangle \\
& H_{2}=\left\langle e_{0}, e_{1}+i e_{2}, e_{6}+i e_{7}, e_{4}-i e_{5}\right\rangle .
\end{aligned}
$$


Remark. Of course over the real numbers, only the first type is relevant: the multiplicative norm on the real Cayley algebra being positive definite, it restricts on any four dimensional subalgebra to a positive definite multiplicative norm, so that any four dimensional subalgebra is a copy of the Hamilton algebra.

\section{The Grassmannian embedding}

\subsection{Orbit structure}

Let $C G$ be the set of four dimensional subalgebras of $\mathbb{O}$. By considering only the imaginary parts of these subalgebras we can consider $C G$ as a closed subvariety of the Grassmannian $G\left(3, V_{7}\right)$, stable under the action of $G_{2}$ (it is closed because the limit of a family of subalgebras is certainly also a subalgebra). We can rephrase the previous discussion in the following way.

Proposition 3.1 The action of $G_{2}$ on $C G$ has only three orbits, consisting of:

1. the orbit $\mathcal{O}_{0} \simeq G_{2} / A_{1}^{s} A_{1}^{l}$ of non degenerate subalgebras, of dimension eight, which is a spherical $G_{2}$-homogeneous space;

2. the orbit $\mathcal{O}_{1}$ of degenerate but non isotropic subalgebras, which is fibered over the adjoint variety of $G_{2}$, the fibers being complements of smooth conics inside projective planes; in particular its dimension is seven;

3. the orbit $\mathcal{O}_{2}$ of isotropic subalgebras, which is isomorphic with the quadric $\mathbb{Q}^{5}$ in $\mathbb{P}\left(V_{7}\right)$.

Proof. The only fact that we have not proved yet is that $H=A_{1}^{s} A_{1}^{l}$ is a spherical subgroup of $G_{2}$. But this already appears in [Kr].

One can check directly that $\mathcal{O}_{0}$ is dense in $C G$, or equivalently that $C G$ is irreducible. We will even show later on that $C G$ is smooth and irreducible.

We will denote by $H$ the orbit closure $\overline{\mathcal{O}}_{1}=\mathcal{O}_{1} \cup \mathcal{O}_{2}$. It admits an equivariant resolution of singularities

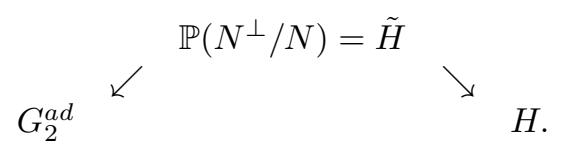

The multiplicative norm descends to the quotient bundle $N^{\perp} / N$, defining a bundle of smooth conics over $G_{2}^{a d}$ whose total space is a hypersurface $E$ inside $\tilde{H}$ which is contracted to the closed orbit $\mathcal{O}_{2}$ in $H$. In particular $H$ is singular along $\mathcal{O}_{2}$, with nodal singularities.

\subsection{The three-form again}

We can also consider $C G$ as a subvariety of the dual Grassmannian $G(4,7)$, by mapping a subalgebra $\mathbb{A}$ of $\mathbb{O}$ to its orthogonal complement $\mathbb{A}^{\perp}$, which is contained in $\operatorname{Im}(\mathbb{O})=V_{7}$. 
Let $T$ denote the tautological rank four vector bundle on $G(4,7)$. By the Borel-Weil theorem, the space of global sections of $\wedge^{i} T^{*}$ is

$$
H^{0}\left(G(4,7), \wedge^{i} T^{*}\right)=\wedge^{i} V_{7}^{*} \text { for } 0 \leq i \leq 4 .
$$

Proposition 3.2 Let $\Omega$ be a generic element of $\wedge^{3} V_{7}^{*}$. The zero locus of the corresponding global section of the rank four vector bundle $\wedge^{3} T^{*}$, is projectively isomorphic to $C G$.

Corollary 3.3 The variety $C G$ is a smooth Fano eightfold with canonical line bundle $K_{C G}=\mathcal{O}_{C G}(-4)$.

Proof. Since the vector bundle $\wedge^{3} T^{*}$ is generated by global section, and since $\Omega$ is generic, the section it defines vanishes, if anywhere, on a smooth eight dimensional subvariety $Z$ of the Grassmannian, invariant under the $G_{2}$-action. By a direct computation it vanishes at the three points defined by $H_{0}, H_{1}, H_{2}$, hence on their $G_{2}$-orbits, hence on the whole of $C G$. This implies that $C G$ is a connected component of $Z$. Finally, we check the connectedness of $Z$ with the help of the Koszul complex

$$
0 \rightarrow \mathcal{O}_{G}(-3) \rightarrow T(-2) \rightarrow \wedge^{2} T(-1) \rightarrow \wedge^{3} T \rightarrow \mathcal{O}_{G} \rightarrow \mathcal{O}_{Z} \rightarrow 0 .
$$

Indeed, a straightforward application of Bott's theorem yields $h^{0}\left(\mathcal{O}_{Z}\right)=1$, which is equivalent to the connectedness of $Z$.

Corollary 3.4 The linear span of $C G$ is $\mathbb{P}\left(S^{2} V_{7}\right) \subset \mathbb{P}\left(\wedge^{3} V_{7}\right)$.

Since $V_{7}$ is self-dual, $S^{2} V_{7}$ embeds equivariantly inside $\operatorname{End}\left(V_{7}\right)$, which can be mapped to $\wedge^{3} V_{7}$ by sending an endomorphism $u$ to the three-form $\Omega(u ., .,$.$) .$ Hence the embedding of $\mathbb{P}\left(S^{2} V_{7}\right)$ inside $\mathbb{P}\left(\wedge^{3} V_{7}\right)$.

Note that $S^{2} V_{7}$ decomposes as the direct sum of a one-dimensional trivial module and an irreducible $G_{2}$-module. In the projectivization of the latter, the closed $G_{2}$-orbit is the quadric $\mathbb{Q}_{5}$ (in its degree two Veronese re-embedding). This is in agreement with our description of the closed orbit $\mathcal{O}_{2}$.

Corollary 3.5 $C G$ is projectively normal in $\mathbb{P}\left(S^{2} V_{7}\right)$, with Hilbert polynomial

$$
P_{C G}(k)=\frac{(k+1)(k+2)^{2}(k+3)}{2880}\left(13(k+2)^{4}+7(k+2)^{2}+4\right) .
$$

In particular the degree of $C G$ is 182 .

Proof. Twisting the Koszul complex by $\mathcal{O}_{G}(k)$ we get

$0 \rightarrow \mathcal{O}_{G}(k-3) \rightarrow T(k-2) \rightarrow \wedge^{2} T(k-1) \rightarrow \wedge^{3} T(k) \rightarrow \mathcal{O}_{G}(k) \rightarrow \mathcal{O}_{C G}(k) \rightarrow 0$.

For $k \geq 0$ the first four vector bundles in this sequence have no higher cohomology, hence $C G$ is projectively normal. For $k=1$, since $\wedge^{3} T(1)=T^{*}$ we get a sequence

$$
0 \rightarrow H^{0}\left(G, T^{*}\right)=V_{7}^{*} \rightarrow H^{0}\left(G, \mathcal{O}_{G}(1)\right)=\wedge^{3} V_{7} \rightarrow H^{0}\left(C G, \mathcal{O}_{C G}(1)\right) \rightarrow 0 .
$$


Since as $G_{2}$-modules $\wedge^{3} V_{7}=V_{7}^{*} \oplus S^{2} V_{7}$ (see [LiE]), we deduce Corollary 3.4. Finally, the Borel-Weil theorem allows us to describe $H^{0}\left(C G, \mathcal{O}_{C G}(k)\right)$ for $k \geq 0$ as an alternate sum of $G L_{7}$-modules whose dimensions are given by the Weyl dimension formula, for example. Therefore we can deduce the Hilbert polynomial $P_{C G}(k)$, and then the degree of $C G$ by looking at its leading term.

In particular there are 119 quadrics vanihing on $C G$. We don't know whether they generate the ideal of $C G$ or not. This would follow, after reduction to positive characteristics, from the Frobenius splitting of a triple product of copies of $C G$, compatibly with its diagonals. Although $C G$ itself, being smooth and spherical, is certainly Frobenius split [BI], we do not know which powers of it inherite this property.

Since $C G$ is spherical, it is also multiplicity free, which means that the decomposition of its homogeneous coordinate ring decomposes into $G_{2}$-modules without multiplicities. We can obtain this decomposition explicitely by using the divisor $H$ and the associated exact sequence

$$
0 \rightarrow \mathcal{O}_{C G}(-1) \rightarrow \mathcal{O}_{C G} \rightarrow \mathcal{O}_{H} \rightarrow 0 .
$$

Twisting by $\mathcal{O}_{C G}(k)$ and taking global sections (recall that $C G$ is Fano with canonical class $\mathcal{O}_{C G}(-4)$, so that $\mathcal{O}_{C G}(k-1)$ has no higher cohomology for any $k \geq 0$ ), we see that it is enough to determine the space of global sections of $\mathcal{O}_{H}(k)$. For this we can pull back to $\tilde{H}$ and make use of its projective bundle structure over the adjoint variety. We deduce that

$$
H^{0}\left(H, \mathcal{O}_{H}(k)\right)=H^{0}\left(G_{2}^{a d}, S^{k}\left(\wedge^{2} N \wedge N^{\perp}\right)^{\vee}\right) .
$$

In order to deduce the latter from the Borel-Weil theorem we need to understand the bundle $\wedge^{2} N \wedge N^{\perp}=\operatorname{det}(N) \otimes N^{\perp} / N$ and the corresponding representation of the parabolic subgroup $P_{\alpha_{2}}$ of $G_{2}$. For that, recall that the adjoint variety $G_{2}^{a d}$ embeds into the Grassmannian $G\left(2, V_{7}\right)$. Moreover $V_{7}$ is generated by the short roots, in particular the highest short root $\theta=2 \alpha_{1}+\alpha_{2}$ coincides with the fundamental weight $\omega_{1}$, while the highest long root $\psi=3 \alpha_{1}+2 \alpha_{2}$ is the fundamental weight $\omega_{2}$. We deduce that the line of highest weight in $G_{2}^{a d}$ corresponds to the plane $N=\left\langle e_{\alpha_{1}+\alpha_{2}}, e_{2 \alpha_{1}+\alpha_{2}}\right\rangle$ in $V_{7}$. Then $N^{\perp} / N=\left\langle e_{-\alpha_{1}}, e_{0}, e_{\alpha_{1}}\right\rangle$, which corresponds to the three-dimensional irreducible representation of the $S L_{2}$ associated with $\alpha_{1}$. Taking its $k$-th power we obtain the direct sum of the irreducible representations of highest weights $(k-2 j) \alpha_{1}$, for $j$ and $k-2 j$ non negative. Since $\alpha_{1}=2 \omega_{1}-\omega_{2}$, we conclude that $S^{k}\left(\wedge^{2} N \wedge N^{\perp}\right)$ is the sum of the irreducible representations of highest weights $(k-2 j) 2 \omega_{1}+2 j \omega_{2}$. We finally get:

Proposition 3.6 The equivariant Hilbert series of $C G$ is

$$
H_{C G}^{G_{2}}(t)=\left(1-t V_{\omega_{0}}\right)^{-1}\left(1-t V_{2 \omega_{1}}\right)^{-1}\left(1-t^{2} V_{2 \omega_{2}}\right)^{-1},
$$

where $\omega_{0}$ denotes the trivial weight. Stated differently,

$$
H^{0}\left(C G, \mathcal{O}_{C G}(k)\right)=\bigoplus_{i+2 j \leq k} V_{2 i \omega_{1}+2 j \omega_{2}} .
$$




\section{Torus fixed points and localization}

Let $T$ be a maximal torus in $G_{2}$.

\subsection{Fixed points and Betti numbers}

Proposition 4.1 The variety $C G$ contains exactly 15 fixed points of $T$.

Proof. The fixed points of $T$ in $C G$ are the intersection points of $C G$ with the projectivized weight spaces of $T$ in $\wedge^{3} V_{7}$. Up to signs and up to the symmetry in $\alpha, \beta, \gamma$ the weights are $2 \alpha$ (six weights of multiplicity one), $\alpha$ (six weights of multiplicity three), $\alpha-\beta$ (six weights of multiplicity one), and 0 (with multiplicity five).

A weight vector of weight $2 \alpha$ is $u_{\alpha} \wedge u_{\beta} \wedge u_{-\gamma}$. The corresponding four dimensional space is $\left\langle u_{0}, u_{\alpha}, u_{\beta}, u_{-\gamma}\right\rangle$, on which the three-form $\Omega$ vanishes identically. We thus get six $T$-fixed points of that type in $C G$.

A basis of weight vectors of weight $\alpha$ is $u_{\alpha} \wedge u_{\beta} \wedge u_{-\beta}, u_{\alpha} \wedge u_{\gamma} \wedge u_{-\gamma}$, $u_{0} \wedge u_{-\beta} \wedge u_{-\gamma}$. No non trivial combination of these vectors is completely decomposable, so the intersection of the projectivized weight space with $G=G\left(3, V_{7}\right)$ reduces to the three corresponding points. But none of these is in $C G$, since they correspond respectively to the four dimensional spaces $\left\langle u_{0}, u_{\alpha}, u_{\gamma}, u_{-\gamma}\right\rangle$, $\left\langle u_{0}, u_{\alpha}, u_{\beta}, u_{-\beta}\right\rangle$ and $\left\langle u_{\alpha}, u_{-\alpha}, u_{-\beta}, u_{-\gamma}\right\rangle$, on which $\Omega$ does not vanish identically.

A weight vector of weight $\alpha-\beta$ is $u_{0} \wedge u_{\alpha} \wedge u_{-\beta}$. The corresponding four dimensional space is $\left\langle u_{\alpha}, u_{-\beta}, u_{\gamma}, u_{-\gamma}\right\rangle$, on which the three-form $\Omega$ vanishes identically. We thus get six new $T$-fixed points of that type in $C G$.

Finally, a basis of the zero weight space is $u_{0} \wedge u_{\alpha} \wedge u_{-\alpha}, u_{0} \wedge u_{\beta} \wedge u_{-\beta}$, $u_{0} \wedge u_{\gamma} \wedge u_{-\gamma}, u_{\alpha} \wedge u_{\beta} \wedge u_{\gamma}, u_{-\alpha} \wedge u_{-\beta} \wedge u_{-\gamma}$. Again it is easy to see that the intersection with $G$ consists in the five corresponding points only. The corresponding four dimensional spaces are $\left\langle u_{\beta}, u_{-\beta}, u_{\gamma}, u_{-\gamma}\right\rangle$ and the two others obtained by permuting $\alpha, \beta, \gamma$, and $\left\langle u_{0}, u_{-\alpha}, u_{-\beta}, u_{-\gamma}\right\rangle$ and its opposite. Among these five only the first three are in $C G$.

In that situation we can use the Byalinicki-Birula decomposition theorem [BB]: if we fix a generic one-dimensional subtorus of $T$, it will again have the

same fixed points as $T$ and the corresponding attracting sets will decompose $C G$ into fifteen strata, isomorphic to affine spaces. The cycle classes of their closures then form a basis of the Chow ring, or of the integer cohomology ring - both are free and isomorphic to each other. By analogy with the case of the usual Grassmannians, we will call these cycle classes Schubert classes.

In order to determine the Betti numbers of $C G$, there remains to compute the dimensions of the strata and count the numbers of strata of each dimension. This is done by listing the weights of the tangent spaces and counting the numbers of negative ones. We get:

Proposition 4.2 The Betti numbers of $C G$ are $1,1,2,2,3,2,2,1,1$. 
For future use we will collect the weights of the tangent bundle to $C G$ at each of its fixed points. To obtain them we simply use the exact sequence

$$
0 \rightarrow T_{C G} \rightarrow T_{G \mid C G}=T^{*} \otimes Q \rightarrow \wedge^{3} T^{*} \rightarrow 0 .
$$

The weights of $T$ and $Q$ are immediate to read at each fixed point.

For simplicity we denote by $(x, y, z)$ the point $\left[u_{x} \wedge u_{y} \wedge u_{z}\right]$. The numbers on the leftmost column of the following table refer to the labelling that we use in the sequel.

$\begin{array}{lll}0 & (\alpha, \beta,-\gamma) & \alpha, \beta, 2 \alpha, 2 \beta, \alpha-\gamma, \beta-\gamma,-\gamma,-\gamma \\ 5 & (\beta, \gamma,-\alpha) & \alpha, \beta, 2 \alpha, 2 \beta, \alpha-\gamma, \beta-\gamma,-\gamma,-\gamma \\ 6^{\prime} & (\gamma, \alpha,-\beta) & 2 \alpha, \alpha, \alpha-\beta,-\beta,-\beta, \gamma-\beta, \gamma, 2 \gamma \\ 3 & (\alpha,-\beta,-\gamma) & -2 \beta,-\beta, \alpha-\beta, \alpha, \alpha, \alpha-\gamma,-\gamma,-2 \gamma \\ 2^{\prime} & (\beta,-\alpha,-\gamma) & -2 \alpha,-\alpha, \beta-\alpha, \beta, \beta, \beta-\gamma,-\gamma,-2 \gamma \\ 8 & (\gamma,-\alpha,-\beta) & -\alpha,-\beta,-2 \alpha,-2 \beta, \gamma-\alpha, \gamma-\beta, \gamma, \gamma \\ 5^{\prime} & (0, \alpha,-\beta) & \alpha, \alpha-\beta, \alpha-\beta,-\beta,-\gamma, \alpha-\gamma, \gamma-\beta, \gamma \\ 2 & (0, \alpha,-\gamma) & -\beta, \alpha-\beta, \alpha, \beta, \alpha-\gamma, \alpha-\gamma, \beta-\gamma,-\gamma \\ 3^{\prime} & (0, \beta,-\alpha) & -\alpha, \beta-\alpha, \beta-\alpha, \beta,-\gamma, \beta-\gamma, \gamma-\alpha, \gamma \\ 1 & (0, \beta,-\gamma) & -\alpha, \beta-\alpha, \alpha, \beta, \alpha-\gamma, \beta-\gamma, \beta-\gamma,-\gamma \\ 6 & (0, \gamma,-\alpha) & \beta, \beta-\alpha,-\alpha,-\beta, \gamma-\alpha, \gamma-\alpha, \gamma-\beta, \gamma \\ 7 & (0, \gamma,-\beta) & \alpha, \alpha-\beta,-\alpha,-\beta, \gamma-\alpha, \gamma-\beta, \gamma-\beta, \gamma \\ 4^{\prime \prime} & (0, \alpha,-\alpha) & -\beta, \alpha-\beta, \beta-\alpha, \beta, \alpha-\gamma, \gamma-\alpha, \gamma,-\gamma \\ 4^{\prime} & (0, \beta,-\beta) & -\alpha, \alpha, \alpha-\beta, \beta-\alpha, \gamma, \gamma-\beta,-\gamma, \beta-\gamma \\ 4 & (0, \gamma,-\gamma) & -\beta,-\alpha, \alpha, \beta, \gamma-\alpha, \gamma-\beta, \alpha-\gamma, \beta-\gamma\end{array}$

Note that given our generic one dimensional torus, we can replace the attractive strata by the repulsive ones. Their closures give two sets of varieties which meet transitively at one or zero points when their dimensions add up to the dimension of $C G$, so that their cohomology classes are Poincaré dual basis of the cohomology ring. We deduce that our Schubert basis is Poincaré self-dual up to a permutation.

Since there is a unique codimension one stratum, we can deduce:

Corollary 4.3 The Picard group of $C G$ is $\operatorname{Pic}(C G)=\mathbb{Z} \mathcal{O}_{C G}(1)$.

The affine cell of maximal dimension has for complement the closure of the codimension one stratum, which is not difficult to analyze. We leave to the reader the proof of the following statement.

Proposition 4.4 Let $\ell \in \mathbb{Q}^{5}$ define an isotropic line in $\operatorname{Im}(\mathbb{O})$. Let

$$
X_{1}(\ell)=\{\mathbb{A} \in C G, A \cap \ell \mathbb{O} \neq 0\},
$$

a special hyperplane section of $C G$. Then the complement of $X_{1}(\ell)$ in $C G$ is an eight dimensional affine space. 
Since, as we will prove below, the cohomology ring is generated by the Schubert classes of degrees one and two, we will complete the picture by describing the codimension two strata of the stratification, or rather their closures, that we denote by $X_{2}$ and $X_{2}^{\prime}$.

Proposition 4.5 There exists $\ell \in \mathbb{Q}^{5}$ such that

$$
X_{2}^{\prime}=X_{2}^{\prime}(\ell)=\{\mathbb{A} \in C G, \operatorname{Im}(\mathbb{A}) \cap \ell \mathbb{O} \neq 0\} .
$$

There exists a null-plane $N$, containing $\ell$, such that

$$
X_{2}=X_{2}(N)=\left\{\mathbb{A} \in C G, \operatorname{dim}\left(\operatorname{Im}(\mathbb{A}) \cap N^{\perp}\right) \geq 2\right\} .
$$

Note that these are both restrictions of special codimension two Schubert cycles on the usual Grassmannian.

\subsection{Equivariant cohomology classes}

Our Schubert classes define a basis of the cohomology ring over $\mathbb{Z}$, and also of the equivariant cohomology ring over the polynomial ring $\Lambda=H_{T}^{*}(\mathrm{pt})=$ $\mathbb{C}\left[\alpha_{1}, \alpha_{2}\right]=\mathbb{C}[\alpha, \beta, \gamma] /\langle\alpha+\beta+\gamma\rangle$. Recall (for example from $[\mathrm{Br}]$ ) that the equivariant cohomology classes can be defined in terms of the GKM-graph $\Gamma$ of the variety $C G$, whose vertices are the $T$-fixed points, and where there is an edge between two vertices when the corresponding $T$-fixed points can be joined by a $T$-equivariant curve. The graph $\Gamma$ is represented below, it has a nice $S_{3} \times \mathbb{Z}_{2}$-symmetry.

Remarks. Although there are finitely many $T$-fixed points in $C G$, it is not true that there are only finitely many $T$-stable curves joining them. It is not difficult to find the exceptions to finiteness: for each boundary triangle of the GKM graph $\Gamma$ there is a $\mathbb{P}^{2}$ covered by the $T$-stable curves joining the points corresponding to the vertices of the triangle; and for each of the three branches of the central star of $\Gamma$ there is a $\mathbb{P}^{1} \times \mathbb{P}^{1}$ covered by $T$-stable curves.

The choice of a generic one-dimensional torus of $T$ breaks the symmetry of the picture, each vertex being put in correspondence with a Schubert variety, obtained as the closure of the attractive set of the associated $T$-fixed point $p$. We will denote the Schubert classes by $\sigma_{i}, \sigma_{i}^{\prime}, \sigma_{i}^{\prime \prime}$ if necessary, where $i$ is the codimension. The correspondence with vertices in the GKM graph is encoded in the next figure, where for example $\sigma_{i}^{\prime}$ corresponds to the vertex labelled $i^{\prime}$, and so on. Note that Poincaré duality is given by the central symmetry of the graph, the three central vertices being fixed. 


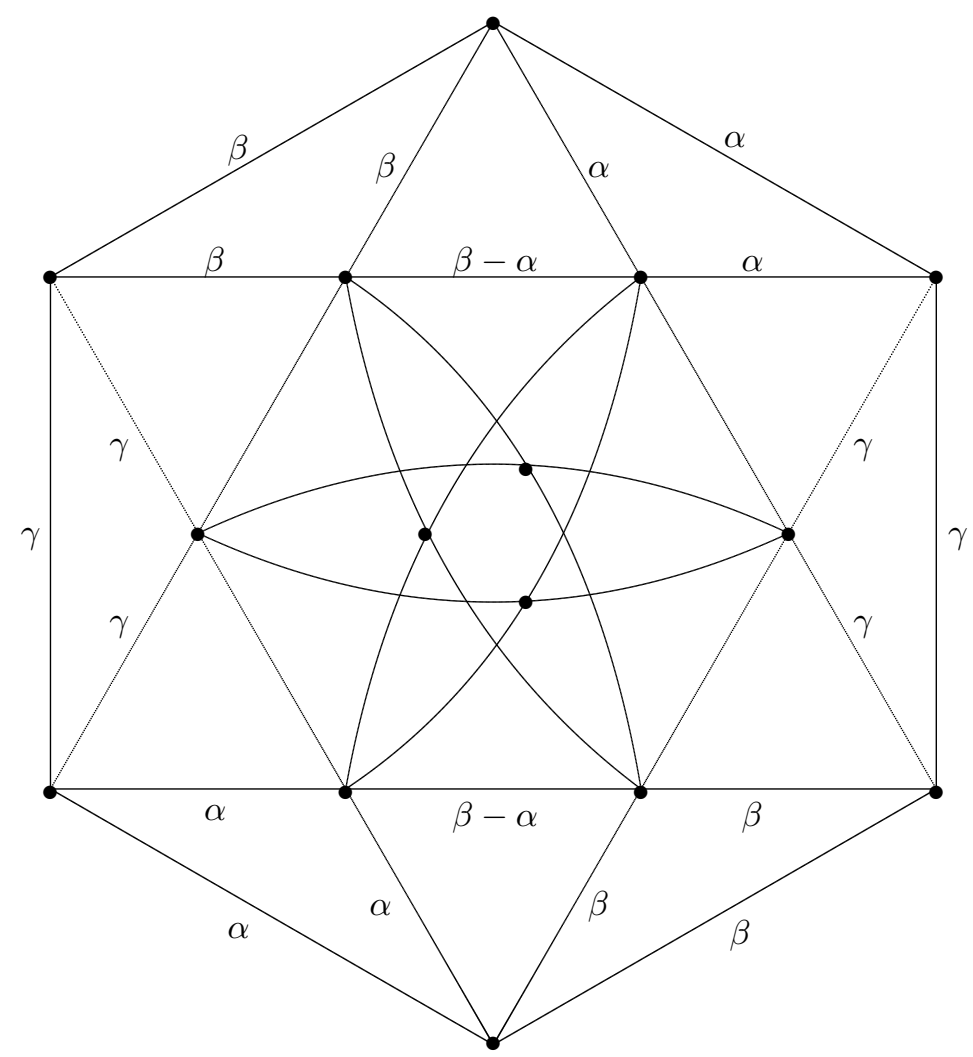

2. The GKM graph of $C G$

Consider a Schubert variety $X$. By the classical localization theorems, its equivariant cohomology class can be represented by a map $f_{X}: \Gamma \rightarrow \Lambda$ with the following properties:

1. each polynomial $f_{X}(q)$ is a homogeneous polynomial, whose degree is equal to the codimension of $X$ in $C G$;

2. $f_{X}(q)=0$ if the $T$-fixed point corresponding to $q$ does not belong to $X$;

3. $f_{X}(p)$ is the product of the weights of the $T$-action on the normal space to $X$ at $p$;

4. suppose that two vertices $q$ and $r$ are joined in $\Gamma$ by an edge, and let $y$ be the weight of the $T$-action on the tangent space at either one of the corresponding $T$-fixed points. Then $f_{X}(q)-f_{X}(r)$ must be divisible by $y$. 
One has more information a priori on the polynomials $f_{X}(q)$ but that turns out to be enough to determine them completely. For example, if $X=C G$, we have $f_{C G}(q)=1$ for any vertex $q$ of $\Gamma$. More interestingly if $X=H$, the unique codimension one Schubert variety, then $f_{H}(q)=\omega(q)-\omega(0)$, where $\omega(q)$ is the weight of the $T$-fixed point $q$ (which is a line in some weight space), and the vertex corresponding to the open strata is denoted by 0 .

Now we use the following inductive strategy. Consider a Schubert variety $X$ of codimension $k$, corresponding to a vertex $p$ of $\Gamma$. Denote by $Y_{1}, \ldots, Y_{m}$ the codimension $k+1$ Schubert varieties contained in $X$, and by $q_{1}, \ldots, q_{m}$ the corresponding vertices (note that $m$ is at most three, and most often two or one). By induction we know the functions $f_{Y_{1}}, \ldots, f_{Y_{m}}$. The function $f_{X}\left(f_{H}-f_{H}(p)\right)$ is homogeneous of degree $k+1$, and is supported on the vertices corresponding to Schubert varieties of codimension bigger than $k$.

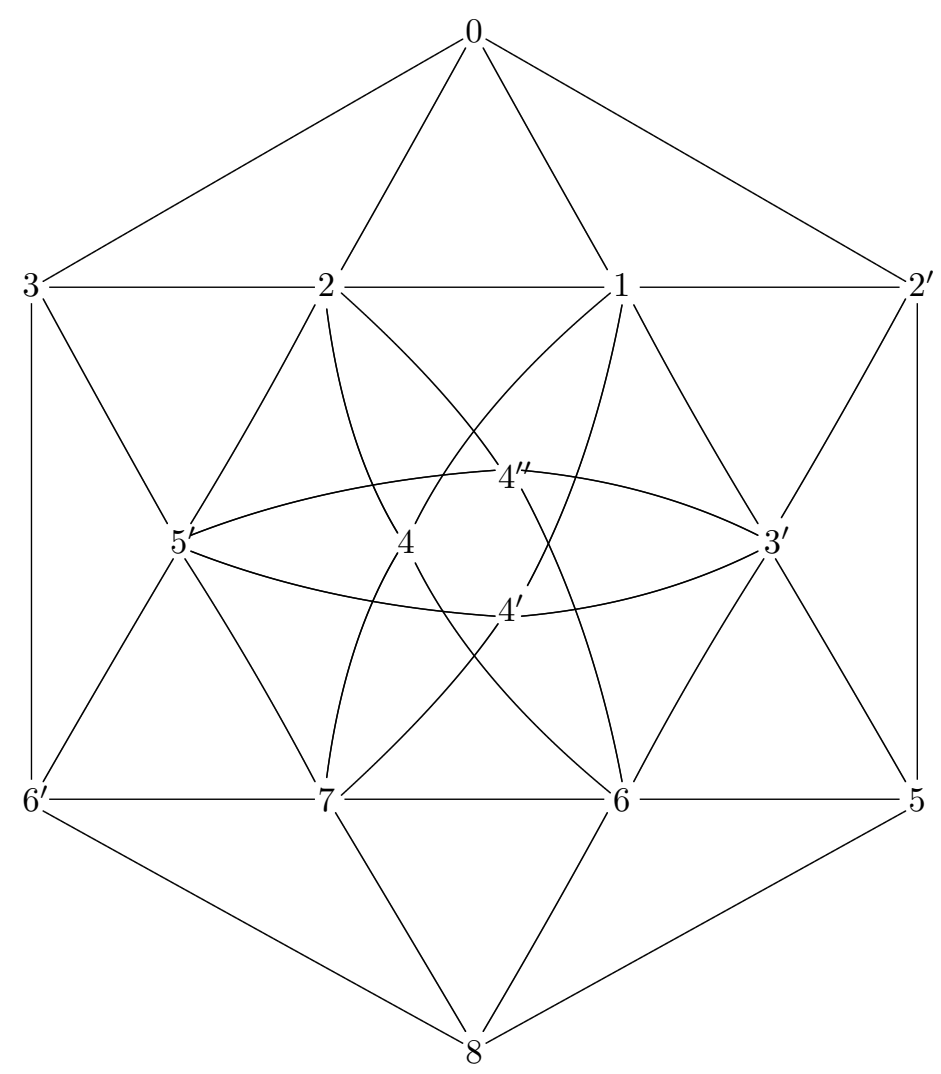

3. Indexing Schubert classes 
The equivariant cohomology class that it represents is then necessarily a combination of the classes of $Y_{1}, \ldots, Y_{m}$ with constant coefficients. Otherwise said, there exists scalars $a_{1}, \ldots, a_{m}$ such that

$$
f_{X}(q)\left(f_{H}(q)-f_{H}(p)\right)=a_{1} f_{Y_{1}}(q)+\cdots+a_{m} f_{Y_{m}}(q)
$$

for each $q$ im $\Gamma$. Letting $q=q_{i}$ we get

$$
f_{X}\left(q_{i}\right)\left(f_{H}\left(q_{i}\right)-f_{H}(p)\right)=a_{i} f_{Y_{i}}\left(q_{i}\right) .
$$

Since $f_{H}\left(q_{i}\right)-f_{H}(p)$ is never zero this determines $f_{X}\left(q_{i}\right)$ up to the constant $a_{i}$. But then the divisibility conditions imposed by the GKM graph allow to determine this constant (in most cases, but not always, it is enough for that to consider $\left.f_{X}\left(q_{i}\right)-f_{X}(p)\right)$. Once we have found $a_{1}, \ldots, a_{m}$, the polynomial $f_{X}(q)$ is completely determined for each $q$.

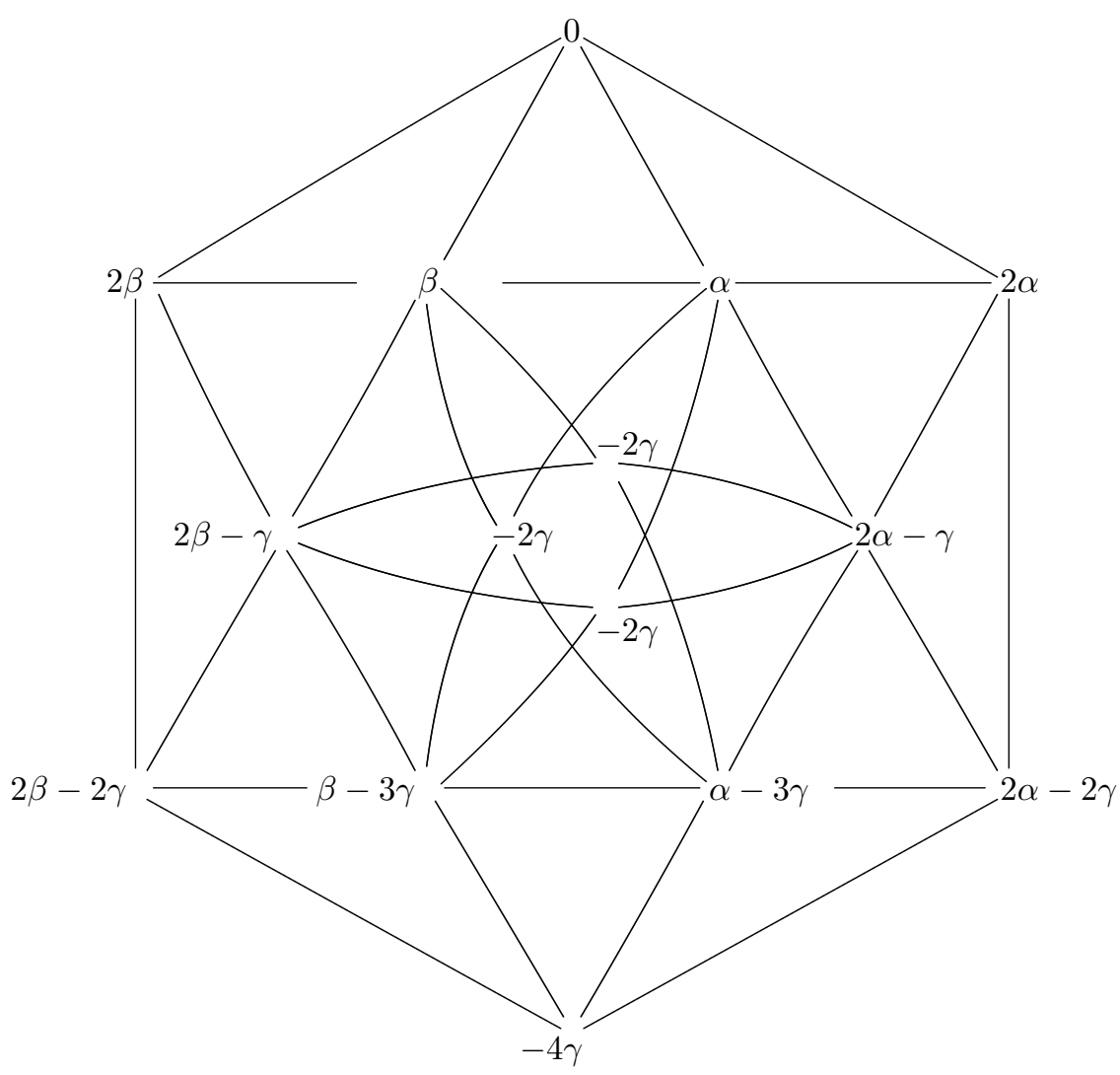

4. The equivariant hyperplane class $\sigma_{1}$ 
Our procedure therefore effectively computes all the equivariant cohomology classes, by descending induction on the degree. For example, we have given below the equivariant class $\sigma_{2}$, which together with the hyperplane class generates the cohomology ring.

Note that along the way we also compute the products of the equivariant Schubert classes by the equivariant hyperplane class. Otherwise said, we get the equivariant Monk formula for $C G$ as a byproduct.

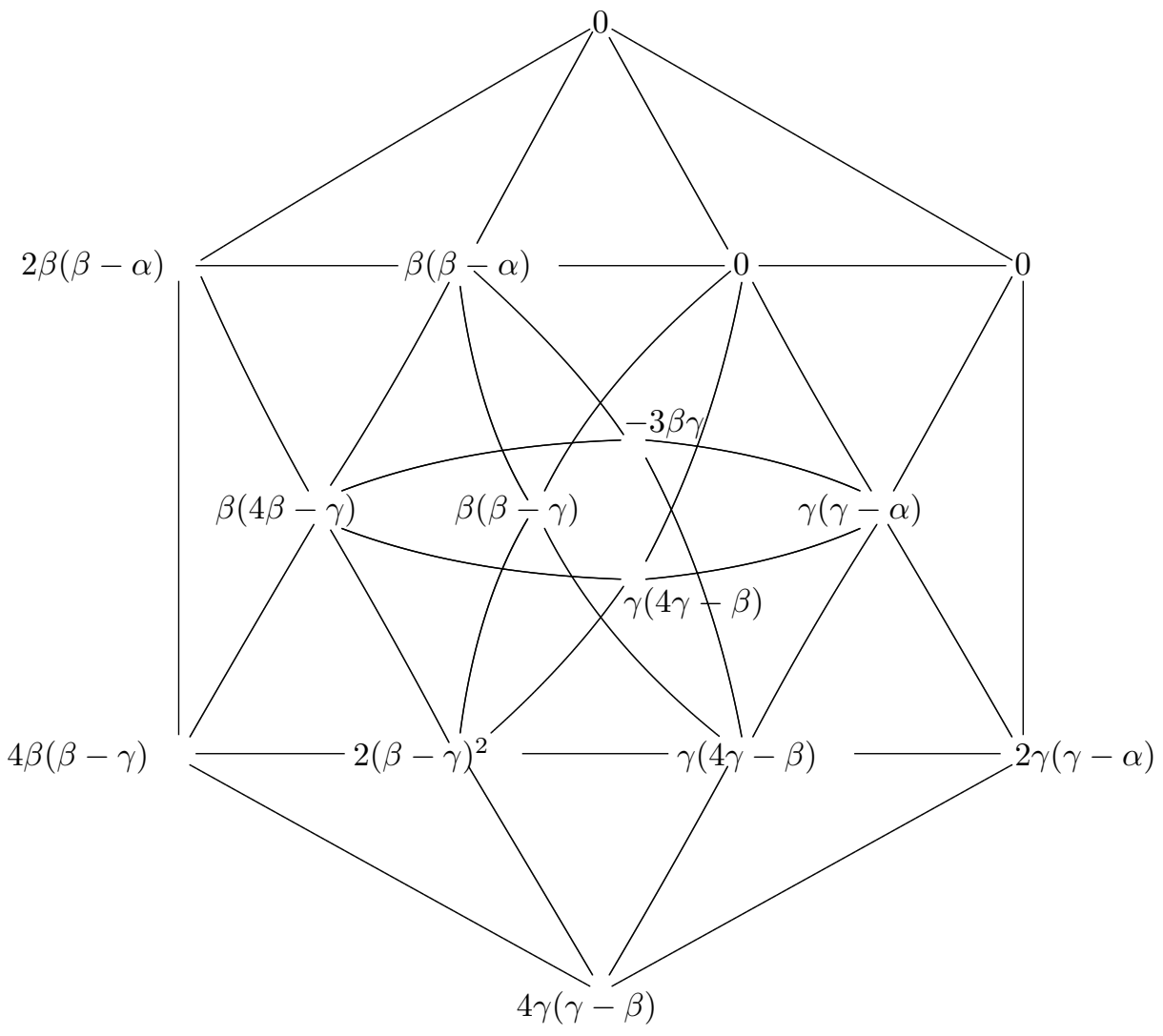

4. The equivariant class $\sigma_{2}$

\subsection{The cohomology ring}

Modding out by non constant homogeneous polynomials we deduce the usual Monk formula, giving the product of a Schubert class by the hyperplane class. We synthetize this formula in the following graph, which we call the Bruhat graph: 


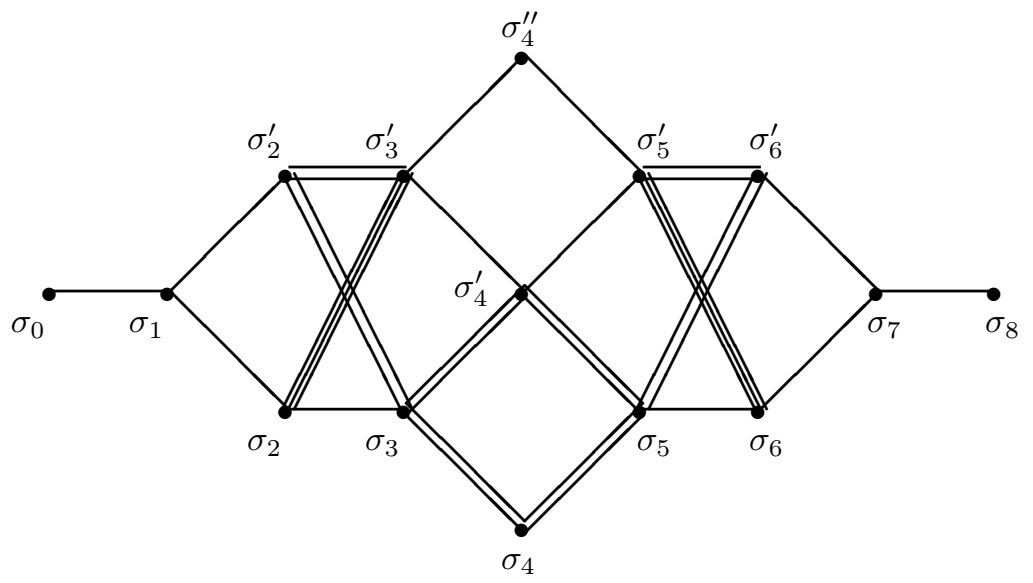

5. The Bruhat graph of $C G$

This must be read as follows: for each Schubert class $\sigma$, the product of $\sigma$ by the hyperplane class is the sum of the Schubert classes connected to it on the column immediately left, with coefficients equal to the number of edges that connect them. (For example $\sigma_{2} H=\sigma_{3}+3 \sigma_{3}^{\prime}$ and $\sigma_{2}^{\prime} H=2 \sigma_{3}+2 \sigma_{3}^{\prime}$.) We can deduce the degrees of all the Schubert classes, that we indicate on the following version of the Bruhat graph. Note that we recover the degree of $C G$ as $182=5^{2}+11^{2}+6^{2}$.

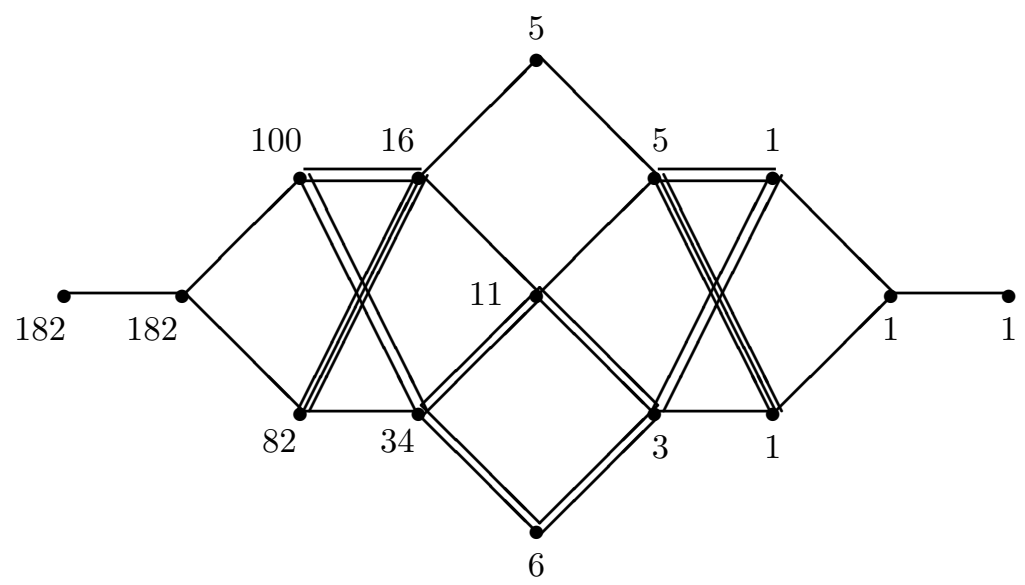

6. The degrees of the Schubert classes

For completeness we compile the other entries of the multiplication table. (The equivariant version could also be derived.) 


$$
\begin{aligned}
\left(\sigma_{2}\right)^{2} & =\sigma_{4}+2 \sigma_{4}^{\prime}+2 \sigma_{4}^{\prime \prime} \\
\sigma_{2}^{\prime} \sigma_{2} & =\sigma_{4}+3 \sigma_{4}^{\prime}+\sigma_{4}^{\prime \prime} \\
\sigma_{3} \sigma_{2} & =3 \sigma_{5}+\sigma_{5}^{\prime} \\
\sigma_{3}^{\prime} \sigma_{2} & =\sigma_{5}+\sigma_{5}^{\prime} \\
\sigma_{4} \sigma_{2} & =\sigma_{6}+\sigma_{6}^{\prime} \\
\sigma_{4}^{\prime} \sigma_{2} & =2 \sigma_{6}+3 \sigma_{6}^{\prime} \\
\sigma_{4}^{\prime \prime} \sigma_{2} & =2 \sigma_{6}+\sigma_{6}^{\prime} \\
\sigma_{5} \sigma_{2} & =\sigma_{7} \\
\sigma_{5} \sigma_{2} & =\sigma_{7} \\
\sigma_{6} \sigma_{2} & =\sigma_{8} \\
\sigma_{6}^{\prime} \sigma_{2} & =0 \\
\left(\sigma_{3}\right)^{2} & =3 \sigma_{6}+5 \sigma_{6}^{\prime} \\
\sigma_{3}^{\prime} \sigma_{3} & =\sigma_{6}+\sigma_{6}^{\prime} \\
\sigma_{4} \sigma_{3} & =2 \sigma_{7} \\
\sigma_{4}^{\prime} \sigma_{3} & =2 \sigma_{7} \\
\sigma_{4}^{\prime \prime} \sigma_{3} & =0 \\
\left(\sigma_{4}\right)^{2} & =\sigma_{8} \\
\left(\sigma_{4}\right)^{2} & =\sigma_{8} \\
\left(\sigma_{4}^{\prime \prime}\right)^{2} & =\sigma_{8}
\end{aligned}
$$$$
\left(\sigma_{2}^{\prime}\right)^{2}=3 \sigma_{4}+3 \sigma_{4}^{\prime}+\sigma_{4}^{\prime \prime}
$$$$
\sigma_{3} \sigma_{2}^{\prime}=5 \sigma_{5}+\sigma_{5}^{\prime}
$$$$
\sigma_{3}^{\prime} \sigma_{2}^{\prime}=\sigma_{5}+\sigma_{5}^{\prime}
$$$$
\sigma_{4} \sigma_{2}^{\prime}=\sigma_{6}+3 \sigma_{6}^{\prime}
$$$$
\sigma_{4}^{\prime} \sigma_{2}^{\prime}=3 \sigma_{6}+3 \sigma_{6}^{\prime}
$$$$
\sigma_{4}^{\prime \prime} \sigma_{2}^{\prime}=\sigma_{6}+\sigma_{6}^{\prime}
$$$$
\sigma_{5} \sigma_{2}^{\prime}=2 \sigma_{7}
$$$$
\sigma_{5} \sigma_{2}^{\prime}=2 \sigma_{7}
$$$$
\sigma_{6} \sigma_{2}^{\prime}=0
$$$$
\sigma_{6}^{\prime} \sigma_{2}^{\prime}=\sigma_{8}
$$

$\left(\sigma_{3}^{\prime}\right)^{2}=\sigma_{6}+\sigma_{6}^{\prime}$
$\sigma_{4} \sigma_{3}^{\prime}=0$
$\sigma_{4}^{\prime} \sigma_{3}^{\prime}=\sigma_{7}$
$\sigma_{4}^{\prime \prime} \sigma_{3}^{\prime}=\sigma_{7}$
$\sigma_{4} \sigma_{4}^{\prime}=0$
$\sigma_{4} \sigma_{4}^{\prime \prime}=0$
$\sigma_{4}^{\prime} \sigma_{4}^{\prime \prime}=0$

We can deduce a presentation of the cohomology ring over $\mathbb{Q}$, choosing the hyperplane class and the codimension two class $\sigma_{2}$ as generators.

Proposition 4.6 The rational cohomology ring of $C G$ is

$$
H^{*}(C G, \mathbb{Q})=\mathbb{Q}\left[\sigma_{1}, \sigma_{2}\right] /\left\langle\sigma_{1}^{5}-5 \sigma_{1}^{3} \sigma_{2}+6 \sigma_{1} \sigma_{2}^{2}, 16 \sigma_{2}^{3}-27 \sigma_{1}^{2} \sigma_{2}^{2}+9 \sigma_{1}^{4} \sigma_{2}\right\rangle .
$$

\subsection{The restriction map}

For future use we need to analyse the restriction of cohomology classes from $G=G(4,7)$ to $C G$. This is rather straightforward and can be done in different ways. In most cases one can simply take a Schubert class on $G$ and compute the degree of its intersection with the fundamental class of $C G$. If the result can be expressed uniquely as a non negative combination of the degrees of the Schubert classes of $C G$ of the same codimension, the relation we get between the degrees is also a relation between the classes, and we are done. When some ambiguity remains, we can compute the intersection of a general Schubert variety on $G$ in the Schubert class of interest, with the Schubert classes on $C G$. Since we know Poincaré duality on $C G$, the result follows.

Proposition 4.7 The restriction map $\iota^{*}: A^{*}(G) \rightarrow A^{*}(C G)$ is given as fol- 
lows, where we denote by $\tau_{\lambda}$ the Schubert class on $G$ defined by a partition $\lambda$ :

$$
\begin{aligned}
& \iota^{*} \tau_{1}=\sigma_{1} \quad \iota^{*} \tau_{3}=\sigma_{3}^{\prime} \quad \iota^{*} \tau_{31}=\sigma_{4}^{\prime}+\sigma_{4}^{\prime \prime} \\
& \iota^{*} \tau_{2}=\sigma_{2}^{\prime} \quad \iota^{*} \tau_{21}=\sigma_{3}+2 \sigma_{3}^{\prime} \quad \iota^{*} \tau_{22}=\sigma_{4}+\sigma_{4}^{\prime}+\sigma_{4}^{\prime \prime} \\
& \iota^{*} \tau_{11}=\sigma_{2} \quad \iota^{*} \tau_{111}=\sigma_{3} \quad \iota^{*} \tau_{211}=\sigma_{4}+2 \sigma_{4}^{\prime} \\
& \iota^{*} \tau_{1111}=\sigma_{4} \\
& \iota^{*} \tau_{32}=\sigma_{5}+\sigma_{5}^{\prime} \quad \iota^{*} \tau_{33}=\sigma_{6}+\sigma_{6}^{\prime} \\
& \iota^{*} \tau_{311}=\sigma_{5}+\sigma_{5}^{\prime} \quad \iota^{*} \tau_{321}=3 \sigma_{6}+3 \sigma_{6}^{\prime} \quad \iota^{*} \tau_{222} \quad=\quad 2 \sigma_{6}+2 \sigma_{6}^{\prime} \\
& \iota^{*} \tau_{221}=3 \sigma_{5}+\sigma_{5}^{\prime} \iota^{*} \tau_{3111}=\sigma_{6}+\sigma_{6}^{\prime} \quad \iota^{*} \tau_{2211}=\sigma_{6}+3 \sigma_{6}^{\prime} \\
& \iota^{*} \tau_{2111}=2 \sigma_{5} \\
& \iota^{*} \tau_{331}=2 \sigma_{7} \quad \iota^{*} \tau_{332}=\sigma_{8} \\
& \iota^{*} \tau_{322}=2 \sigma_{7} \quad \iota^{*} \tau_{3311}=\sigma_{8} \\
& \iota^{*} \tau_{3211}=2 \sigma_{7} \quad \iota^{*} \tau_{3221}=\sigma_{8} \\
& \iota^{*} \tau_{2221}=2 \sigma_{7} \quad \iota^{*} \tau_{2222}=\sigma_{8}
\end{aligned}
$$

Corollary 4.8 The image of $\iota^{*}$ is a sublattice of $A^{*}(C G)$ of index 16 .

\subsection{The projective dual of $C G$}

As a byproduct of our computations we can derive interesting information on the projective dual of $C G$, which is the variety $C G^{\vee}$ parametrizing, in the dual projective space, the tangent hyperplanes to $C G$. We will use the Katz-Kleiman formula [GKZ], following which we should consider the polynomial

$$
c_{C G}(q)=\sum_{i=0}^{8} q^{i+1} \int_{C G} c_{8-i}\left(\Omega_{C G}\right) \sigma_{1}^{i} .
$$

Then, if $c_{C G}^{\prime}(1) \neq 0$, the dual variety of $C G$ is a hypersurface of precisely that degree.

Proposition 4.9 The projective dual variety $C G^{\vee}$ is a hypersurface of degree 17 in $\mathbb{P}^{28}$.

Proof. Having computed the weights of the tangent spaces at the $T$-fixed points, we immmediately deduce the equivariant total Chern class of the tangent bundle $T_{C G}$. A routine computation then yields

$$
\begin{aligned}
& c_{1}\left(T_{C G}\right)=4 \sigma_{1}, \\
& c_{2}\left(T_{C G}\right)=9 \sigma_{2}+7 \sigma_{2}^{\prime}, \\
& c_{3}\left(T_{C G}\right)=28 \sigma_{3}+52 \sigma_{3}^{\prime}, \\
& c_{4}\left(T_{C G}\right)=49 \sigma_{4}+88 \sigma_{4}^{\prime}+46 \sigma_{4}^{\prime \prime}, \\
& c_{5}\left(T_{C G}\right)=76 \sigma_{5}+160 \sigma_{5}^{\prime}, \\
& c_{6}\left(T_{C G}\right)=133 \sigma_{6}+151 \sigma_{6}^{\prime}, \\
& c_{7}\left(T_{C G}\right)=90 \sigma_{7}, \\
& c_{8}\left(T_{C G}\right)=15 \sigma_{8} .
\end{aligned}
$$


Using our computations of the degrees of the Schubert classes we deduce that $c_{C G}(q)=15 q-90 q^{2}+344 q^{3}-860 q^{4}+1492 q^{5}-1784 q^{6}+1438 q^{7}-738 q^{8}+182 q^{9}$. This gives $c_{C G}^{\prime}(1)=17$ and the result follows.

\subsection{Concluding remarks}

The cohomology of $C G$ looks very much like that of the Grassmannian $G(2,6)$, which has the same Betti numbers and a similarly looking presentation, with relations in degree 5 and 6 . An important difference between the two varieties is that the index of $C G$ is four, while the index of $G(2,6)$ is six. This makes much harder the task of computing the quantum cohomology ring of $C G$, since we have much more freedom in the possible quantum deformations of the two relations than in the case of $G(2,6)$ (recall that for any prime Fano manifold, the degree of the quantum parameter is equal to the index). Moreover the fact that $C G$ is only quasi-homogeneous, not homogeneous, makes the computation of the Gromov-Witten invariants much less straightforward since they are not clearly enumerative. We plan to tackle these issues in a sequel to the present paper.

\section{References}

[Baez] Baez J., The octonions, Bull. AMS 39 (2002), no. 2, 145-205.

[BB] Bialynicki-Birula A., Some theorems on actions of algebraic groups, Annals of Math. (2) 98 (1973), 480-497.

[Br] Brion M., Equivariant cohomology and equivariant intersection theory, in Representation theories and algebraic geometry (Montreal 1997), 1-37, Kluwer 1998.

[BI] Brion M., Inamdar S.P., Frobenius splitting of spherical varieties, in Algebraic groups and their generalizations: classical methods, 207-218, Proc. Sympos. Pure Math. 56, AMS 1994.

[GKZ] Gel'fand I.M., Kapranov M.M., Zelevinsky A.V., Discriminants, resultants, and multidimensional determinants, Birkhäuser 1994.

[Kr] Krämer M., Sphärische Untergruppen in kompakten zusammenhängenden Liegruppen, Compositio Math. 38 (1979), no. 2, 129-153.

[LM1] Landsberg J.M., Manivel L., The projective geometry of Freudenthal's magic square, J. Algebra 239 (2001), no. 2, 477-512.

[LiE] LiE, A computer algebra package for Lie group computations, available at http://young.sp2mi.univ-poitiers.fr/ marc/LiE/.

Institut de Mathématiques de Marseille, UMR 7373 CnRS/AiX-Marseille Université, Technopôle Château-Gombert, 39 rue Frédéric Joliot-Curie, 13453 MARSEILle Cedex 13, France

Email address: laurent.manivel@math.cnrs.fr 